\title{
ACUTE ADRENAL INSUFFICIENCY
}

\author{
By Paul Fourman, M.D., M.R.C.P. \\ Nuffield Department of Clinical Medicine, University of Oxford
}

In acute adrenal insufficiency we are faced with the interaction of many factors ; if in trying to disentangle them I have cut some knots it is for the sake of brevity.

Acute adrenal insufficiency may result from sudden loss of adrenal function by haemorrhage, thrombosis or ablation. More often it occurs in a patient with chronic adrenal insufficiency, either in the natural course of the illness or following an injury such as infection, operation or exposure. Acute adrenal insufficiency is characterized by prostration and collapse with low blood pressure and rapid pulse. It is usually accompanied by gastro-intestinal symptoms : anorexia, vomiting and diarrhoea, and sometimes abdominal pain and hiccup. Headache is common. The patient is at first weak and apathetic but he may become restless and confused, sometimes with high and swinging fever, before he finally lapses into a fatal coma.

Now, in patients with intact adrenal glands who have been exposed to different types of injury or "stress" there is, underlying the specific response to each type of injury, a general response common to all. This is the alarm reaction of Selye and is partly a result of discharge of adrenal hormone. In the alarm reaction adrenal hormone is liberated in large amounts and is probably rapidly consumed by the tissues. In Addisonian patients the alarm reaction cannot occur, but the response to stress again follows a general pattern which we know as an adrenal crisis, so that in Addisonian patients too there is a non-specific response to injury. Presumably the reaction to injury of patients with intact adrenals combines the effects of a discharge of adrenal hormone and another mechanism which is unmasked in adrenal insufficiency. This other mechanism should account for the rapid utilization of adrenal hormone in the alarm reaction. What this mechanism is we do not know. Thyroid is one hormone that may increase the need for adrenal hormone, for thyroid may precipitate adrenal crisis in patients with hypopituitarism ; apart from this, adrenal crisis is less common in patients whose adrenal insufficiency is due to hypopituitarism than in patients with Addison's disease.

Addison's disease is characterized by a failure to conserve sodium and we usually think of the crisis of acute adrenal insufficiency as a condition of shock brought about by salt depletion. This might be true in a patient with Addison's disease who is in crisis when he first presents ; in him the crisis is the climax of a long illness during which there has been time for the sodium stores to become depleted. The sodium depletion may represent a stress that released the crisis rather than the immediate cause of the shock-like state.

Sodium depletion is not an essential feature of adrenal crisis. Adrenal crisis can develop in a matter of hours, but it takes a few days for the loss of sodium to produce a serious depletion. If the adrenal insufficiency has arisen suddenly depletion of sodium has not had time to develop; if the crisis has arisen in a patient already under treatment for Addison's disease depletion of sodium will have been prevented by the previous treatment. These patients may show little biochemical evidence of acute adrenal insufficiency beyond hypoglycaemia, and even this may be lacking. In our ignorance we take refuge in saying that stress has produced an increased demand for adrenal hormone to which the adrenal glands are unable to respond.

It is important to distinguish between cases with and without salt depletion, for their treatment will differ. In both instances the patients will need an active preparation of adrenal hormone to control collapse and hypoglycaemia, and the greater the precipitating stress the greater the demand for hormone ; but the one group will also need to be given salt and may need to be helped to conserve salt ; the other may be killed by an excess of salt and salt-retaining hormone, for the patient with adrenal insufficiency can no more excrete salt in the face of excess than conserve it in the face of depletion. 
It is possible that there is only one adrenal hormone in nature, but in practice among the artificial hormone preparations that are available to us two important actions are divorced. The first is to conserve sodium and discharge potassium ; the second is to control glycogen metabolism and prevent hypoglycaemia-associated with this second action is the ability to resist stress. The two types of preparation are represented by the artificial compound deoxycortone acetate (DCA) on the one hand, and by cortisone on the other. Whole adrenal extract probably combines both actions, but compared to the whole extract DCA has an exaggerated effect on sodium retention.

DCA has very little action other than to restore electrolyte balance, but if the balance is upset this action may be life-saving. The main action of deoxycortone on electrolytes is to cause retention of sodium. It cannot retain sodium unless sodium is given and the effect depends not so much on the dose of deoxycortone as on the amount of sodium that the patient is receiving. DCA has another effect on electrolyte balance, to cause a discharge of accumulated potassium from the body. This effect is greater, the greater the dose of DCA and is excessive if $\mathrm{DCA}$ is given in excess.

DCA does not bear much relation to the hormone that is normally called forth and rapidly consumed in stress. This is more nearly .represented by the whole extract of the gland, and nowadays by cortisone, and perhaps in the future by hydrocortisone (compound F) if it should be economically synthesized. These preparations cduse a discharge of potassium but have only minor effects on sodium. We know that this type of hormone will prevent the hypoglycaemia of adrenal insufficiency, but this is not enough to explain why it should be essential to survival in the face of stress such as exposure, operation, or infection. It is hardly possible to give an overdose of adrenal extract, but an excess of DCA will limit the amount of cortisone that can safely be given, owing to the risk of potassium deficiency.

Adrenal crisis is an emergency during which the patient may at any moment die ; treatment must be prompt. In treatment our aim is :

I. To treat the shock.

2. To remove the stress.

3. To replenish sodium and give sugar.

4. To supply adrenal hormone as required, and 5. Not to kill the patient.

This may be elaborated :

I. Keep the patient warm and disturb him as little as possible - even a blanket bath may be lethal. Morphia is dangerous. Chart the pulse and the blood pressure, at first hourly and later every four hours, as in a patient with shock.

2. Look for any infection and use antibiotics freely. Fever is no guide to whether or not these patients have an infection.

3. If the patient is depleted of sodium, give $5 \stackrel{\curvearrowright}{\circledR}$ per cent. glucose in normal saline intravenously, $C$ but not more than 3 litres in 24 hours. Stop the $\overrightarrow{\vec{F}}$ drip as soon as possible after the patient begins to take by mouth. Control the treatment by following the serum sodium and the haematocrit. Watch for $\frac{\bar{c}}{\bar{s}}$ any sign of overloading with fluid. If the patient $\frac{\sigma}{\sigma}$ is not depleted of sodium, very little saline may be $\stackrel{\varnothing}{\circ}$ needed; give ro per cent. glucose in water intra- के venously and glucose by mouth as soon as possible. $\overrightarrow{0}$ Control the treatment by the blood glucose. Here the intake of fluid should not exceed 2 litres in the $\vec{\omega}$ first 24 hours and thereafter depends on the $\frac{\rho}{8}$ urinary output. An intake of $\mathrm{I}$ litre in excess of $\frac{0}{7}$ the urinary output is safe. Do not give large doses of DCA routinely. If needed at all, 10 $\mathrm{mg}$. त़ or less may suffice on the first day and $5 \mathrm{mg}$. or less on succeeding days.

4. Be extravagant with whole adrenal extract. $\frac{N}{G}$ Give 20 or $40 \mathrm{cc}$. into the drip and then ro cc. $\mathrm{G}$ every hour until the systolic blood pressure is $\frac{}{5}$ $80 \mathrm{~mm}$., and every 2 hours until nausea, vomiting, $\vec{\rightarrow}$ abdominal pain and diarrhoea have stopped.

With cortisone it is possible to use less adrenal extract than hitherto but, because the extract can $\overrightarrow{0}$ be given intravenously and cortisone cannot, of still rely on adrenal extract for-a rapid effect. Two milligrams of cortisone are equivalent to 10 c.C. of extract. Cortisone acts more rapidly by mouth than intramuscularly, but if the patient cannot swallow, cortisone must be given intramuscularly, 응 say 100 or $200 \mathrm{mg}$. if no excess of DCA has been $\stackrel{2}{\overrightarrow{7}}$ given. As soon as the patient can take by mouth, $\frac{\vec{O}}{3}$ give $50 \mathrm{mg}$. followed by $25 \mathrm{mg}$. every 6 hours. When symptoms have receded and for 3 days after that, the patient should have either extract, Io c.c. every 4 hours, or cortisone $12.5 \mathrm{mg}$. every 6 hours. $\frac{3}{3}$ The patient is then ready for maintenance therapy and a small dose of cortisone, say 12.5 or $25 \mathrm{mg}$. 3 . daily, can be given partly to replace DCA. The cortisone may be given by mouth in four divided doses daily, in a mixture, or as an intramuscular 0 injection of 50-100 mg. twice a week. These dosage schemes provide only a rough guide. In $\frac{D}{0}$ particular the patient may require more adrenal hormone if there is persistent infection or other $N$ stress such as operation.

Some patients have a very stormy course and the blood pressure fails to rise. Infusions of plasma ${ }^{\omega}$ may be added to the treatment. Injections of adrenaline $(0.3 \mathrm{mg}$.) have been recommended but $\stackrel{\circ}{\mathrm{C}}$ may have an undesirable effect in increasing the pulse rate. Nor-adrenaline may have a place in ${ }_{7}^{+}$ the treatment of the hypotension, particularly to prevent the sudden collapse produced by adrenal surgery. It is given in a drip at a rate of $5-35 \frac{\stackrel{?}{\mathrm{Q}}}{\circ}$ 
micrograms a minute, with careful watch on the blood pressure.

The absolute eosinophil count may be a helpful guide to treatment but probably only after the first emergency is over. It is reasonable to aim at a count below 50 per cu. $\mathrm{mm}$. in the presence of stress.

5. The patient may be killed by an excess of salt, fluid or DCA ; the venous pressure does not give warning of this. Autopsy may reveal pleural and pericardial effusions when there was no rise of vẹnous pressure in life.

Chronic adrenal insufficiency is usually due to destruction or atrophy of the adrenal glands but it may complicate hypopituitarism and it may accompany adreno-genital virilism. In all these diseases cortisone has a rational place in maintenance treatment as well as in the treatment of crisis. Patients with Addison's disease need much less cortisone than patients with rheumatoid arthritis ; tuberculosis is probably not aggravated when cortisone is given in these physiological doses. Appetite improves, strength returns and mental powers increase. Hypoglycaemia is controlled. The risk of sudden crisis and death is diminished.

Acute adrenal insufficiency can be forestalled if one recognizes that any stress such as hard physical work, infection or operation leads to an increased demand for hormone. In a patient who cannot respond to this demand because he lacks adrenal glands large amounts of adrenal extract or cortisone should be given. Patients with adrenal atrophy secondary to hypopituitarism or with unilateral atrophy due to a contralateral tumour which is to be removed present a special case ; in them the adrenal glands may be stimulated with adreno-corticotropic hormone.

\section{CORRESPONDENCE}

\section{REQUEST FOR REPRINTS CONCERNING STRESS AND THE ADAPTIVE HORMONES}

DEAR SIR,

In perusing the current literature with which this journal is concerned, we note that an everincreasing number of its articles deals with problems pertaining to research on 'stress' and the so-called 'adaptive hormones' (ACTH, STH, corticoids, adrenergic substances, etc.).

We are writing you because, in our opinion, the success of research in this complex and rapidly developing field largely depends upon the prompt availability and evaluation of relevant publications, a task for which we should like to solicit the assistance of your readers.

In $195^{\circ}$ our Institute initiated the publication of a series of reference volumes entitled 'Annual . Reports on Stress' (Acta Medical Publishers, Montreal) in which the entire current world literature is surveyed every year (usually between 2,000 and 4,000 publications). Up to now we have had to compile the pertinent literature partly from medical periodicals, monographs, abstract journals and partly from reprints sent to us by the authors themselves. Of all these, reprints proved to be the best source of data which we felt deserved prompt attention in our annual reports. Hence, in the past, we have sent out several thousand individual reprint requests to authors of whom we knew that they are currently engaged in research on stress and allied topics. Even this procedure did not give us the wide coverage which would be desirable, because it is materially impossible to contact all these authors individually and it often takes too much time to get the requested reprints.

It is evident that in order to ensure prompt inclusion of publications in the annual reports, these surveys must develop into a co-operative effort between the authors of original papers and the reviewers. This co-operation has been greatly enhanced of late by the publication of announcements, in several medical journals, encouraging investigators interested in stress research to send us their reprints for this purpose as soon as they become available.

We should be grateful if by the publication of this note you would also bring this problem to the attention of your readers.

We are, Sir;

Very sincerely yours,

Hans Selye, M.D., Ph.D., D.Sc., F.R.S.(C.). Professor and Director of the Institute of Experimental Medicine and Surgery.

Alexander Horava, M.D.,

$$
\begin{gathered}
\text { Co-author of the 'Annual Reports on } \\
\text { Stress.' }
\end{gathered}
$$

